\title{
DERMATOFIBROSARCOMA PROTUBERANTE CERVICAL: RELATO DE CASO
}

\section{ARTIGO ORIGINAL}

NETO, Alonso Alves Pereira ${ }^{1}$

ESPÓSITO, Mario Pinheiro ${ }^{2}$

OLIVEIRA, Ronan Djavier Alves ${ }^{3}$

PASSOS, Fabio Manoel dos ${ }^{4}$

PLACHESKI, Ana Carolina Galindo ${ }^{5}$

PELIZER, Carlos Antônio Albuquerque ${ }^{6}$

ESPÓSITO, Guilherme Soriano Pinheiro ${ }^{7}$

\footnotetext{
${ }^{1}$ Residente em Otorrinolaringologia e Cirurgia Cérvico-Facial, Graduado em Medicina pela União das Faculdades dos Grandes Lagos - UNILAGO.

2 Doutor em Otorrinolaringologia pela Faculdade de Ciências Médicas da Santa Casa de São Paulo.
}

${ }^{3}$ Residente em Otorrinolaringologia e Cirurgia Cérvico-Facial, Graduado em Medicina pela Universidade de Cuiabá - UNIC.

${ }^{4}$ Residente em Otorrinolaringologia e Cirurgia Cérvico-Facial; Graduado em Medicina pela Universidade Federal do Paraná - UFPR.

${ }^{5}$ Residente em Otorrinolaringologia e Cirurgia Cérvico-Facial; Graduada em Medicina pela Universidade de Cuiabá - UNIC.

${ }^{6}$ Residente em Otorrinolaringologia e Cirurgia Cérvico-Facial; Graduado em Medicina pelo Centro Universitário São Lucas - UNISL.

${ }^{7}$ Residente em Otorrinolaringologia e Cirurgia Cérvico-Facial; Graduado em Medicina pelo Centro Universitário São Lucas - UNISL. 
NETO, Alonso Alves Pereira. Et al. Dermatofibrosarcoma Protuberante Cervical: Relato de caso. Revista Científica Multidisciplinar Núcleo do Conhecimento. Ano 05, Ed. 08, Vol. 16, pp. 97-106. Agosto de 2020. ISSN: 2448-0959, Link de acesso: https://www.nucleodoconhecimento.com.br/saude/dermatofibrosarcoma

\section{RESUMO}

A dermatofibrosarcoma protuberante é entendido como um tumor dermatológico raro, que se manifesta, clinicamente, como nódulos protuberantes na pele, de crescimento lento, mas localmente agressivo, podendo invadir o tecido subcutâneo, os músculos, entre outras partes moles, com alto índice de recidiva, e baixa taxa de metástase. Este tumor é comumente encontrado em troncos e membros, no sexo masculino, entre a terceira e quinta década. O diagnóstico é realizado pela anamnese, exame físico e de imagem, e exame anatomopatológico. A conduta é essencialmente cirúrgica, com excisão de margem ampla de aproximadamente 3 centímetros, e ressecção tridimensional, sendo a radioterapia adjuvante e reoperação, eventualmente, necessária. Relata-se um caso, de uma paciente de 59 anos, atendida no Hospital do Câncer do Mato Grosso, com dermatofibrosarcoma protuberans cervical à esquerda, apresentando-se como nódulos protuberantes cervicais inespecíficos e avermelhados; a região afetada é pouco comum, e localmente agressivo. Realizada cirurgia de ressecção tumoral, e acompanhamento clínico e por imagem com equipe de otorrinolaringologia, cirurgia de cabeça e pescoço, e oncologia; sem recidiva já há 3 anos, e até o momento sem necessidade de radioterapia. É necessário salientar a necessidade de se obter um diagnóstico correto desta neoplasia, se possível precoce, uma correta orientação cirúrgica como descrevem as literaturas, além de um adequado acompanhamento pós-operatório, para evitar a recorrência do tumor, que caso ocorra, seja prontamente tratada.

Palavras-Chave: Dermatofibrosarcoma protuberans, tumor dermatológico, nódulos protuberantes cervicais, ressecção tumoral. 


\section{INTRODUÇÃO}

O dermatofribrasarcoma protuberans (DFSP) ou dermatofibrasarcoma protuberante é considerado como um tumor maligno, que se desenvolve, tendenciosamente, como nódulos salientes.

Este tumor não é encontrado com tanta frequência, sendo restrito à incidência de " 0,8 a cinco casos por milhões de habitantes/ano, correspondendo a menos de $0,1 \%$ de todas as malignidades e a cerca de $1 \%$ dos sarcomas de partes moles", como afirma Diniz; Ramos e Nunes (2013, n.p.). É notório salientar, que em vários estudos se tomou conhecimento da similaridade em relação ao acometimento em ambos os sexos, entretanto, há maior ascendência em homens e torna-se frequente em até quatro vezes mais, relacionando-se à idade dos pacientes. Esse tumor é mais propenso a ser acometido em pacientes idosos. A literatura é indicada que a faixa etária mais acometida pelo DFSP é dos 30 aos 50 anos, e a sua localização se dá no tronco (40-60\%), depois os membros (20-30\%) e, por fim, de cabeça e pescoço (10$15 \%)$.

Em estudos recentes, é possível visualizar caso não haja um trauma que anteceda o tumor, a sua descrição na literatura, se faz em 10 a $20 \%$ em relação aos casos, mas se houver trauma, a descrição de desenvoltura se dá em cicatrizes de cirurgia, queimaduras, imanização para varicela ou BGG, sendo o seu potencial de crescimento maior durante a gravidez, devido aos receptores de progesterona no tumor.

O tumor, inicialmente, em tronco e em membros pode aparecer de forma a ser confundido como tumor benigno, clinicamente. Apresentando-se como um nódulo assintomático, mas, caso não haja um tratamento, pode evoluir de tamanho $\mathrm{e}$, consequentemente, sofrer o processo de ulceração. A caracterização do tumor é tomada por uma coloração avermelhada ou amarronzada, podendo-se ser encontrada no início ou como evolução de tumor em áreas de atrofia, induração ou mesmo, telangiectasia. O crescimento do DFSP se dá de forma lenta e infiltrativo, dentro da 
literatura, se compreende como uma alta taxa de reincidir nos locais, entretanto, há uma baixa taxa de haver metástase.

Histologicamente, o DFSP se dá a partir de células fusiformes, como uma coleção densa uniforme, arranjando-se como um estoriforme. Ao se infiltrar no subcutâneo, o tumor distorce a forma do tecido adiposo, assemelhando-se à aspecto de favo de mel. Por intermédio do microscópio, haverá uma avaliação de margens clínicas, em que se espalhará localmente na derme, no tecido subcutâneo e no tecido muscular.

De acordo com a literatura, a forma padrão de tratamento para o tumor DFSP se faz com uma excisão extensa com local em que se encontra o tumor, incluindo-se a fáscia subjacente e margens com tecido de aparência normal, em todos os planos. Em relação as margens cirurgias, a literatura recomenda que se faça uma incisão de pelo ao menos $2 \mathrm{~cm}$, preferivelmente, $3 \mathrm{~cm}$ de tecido sadio, a partir da distância entre a margem macroscópica do tumor, tendo como base a ressecção tridimensional, que é composta por pele, tecido subcutâneo e fáscia subjacente. A margem cirúrgica negativa, para alguns autores, não é suficientemente boa, caso haja uma reoperação, a margem histológica indicada seria menor que $1,5 \mathrm{~cm}$. Na cirurgia, pode haver dificuldades na remoção cirúrgica, já que, a projeção profunda em direção a fáscia e o músculo dificultam a delimitação.

O tratamento padrão do DFSP, defendido durante décadas dentro do âmbito literário se dá por meio da excisão cirúrgica radical, com margens laterais ampliadas que contenham tecidos sadios, isso se dá devido ao comportamento progressivo e assíduo da neoplasia.

De acordo com o artigo Dermatofibrosarcoma protuberans: série de 27 casos consecutivos (2016),

a radioterapia administrada depois da cirurgia pode reduzir significativamente o risco de recorrência local em pacientes que apresentam ou são propensos a ter margens fechadas ou positivas. Tem provado benefício em radioterapia adjuvante no pós-operatório em tumores com excisão incompleta. (CAPPELLINA et al., 2016, n.p.) 
Há alguns estudos que inferem que o tratamento disposto com a radioterapia possa parecer controverso. Para sarcomas de baixo grau $(>5 \mathrm{~cm})$, é preconizado o uso da radioterapia adjuvante e para sarcomas de alto grau $(<5 \mathrm{~cm})$, só quando há margens cirúrgicas são positivas, próximas ou mesmo, para tumores que não possam ser excisado. A radioterapia será utilizada para tratamentos de tumores não estabelecidos e a quimioterapia ganha destaque de indicação apenas quando há metástase.

\section{RELATO DE CASO}

Paciente C. L. A. R. de 59 anos, sexo feminino, vem ao consultório em outubro de 2009, queixando-se de nódulo em região cervical esquerda, há oito meses de crescimento progressivo, indolor, e que a seis meses apresentou hiperemia local. Exame da cavidade oral sem alterações e exame do pescoço com presença de área de espessamento em região cervical esquerda em nível III, indolor, aderido ao músculo esternocleidomastoideo esquerdo, sem linfadenite palpável. A paciente nega hipertensão arterial, diabetes mellitus e tabagismo. A mesma, foi submetida a ressecção de nódulo de $2,2 \mathrm{~cm}$ de diâmetro com margens comprometidas. anatomopatológico revelou Dermatofibrosarcoma protuberans e na tomografia cervical, infiltração de pele e tecido muscular estriado e infiltração perineural presente. Videolaringoscopia sem alterações. A cirurgia de ressecção de partes moles no lado esquerdo do pescoço com margem de $3 \mathrm{~cm}$ foi realizada um mês após a data da primeira consulta. Após a cirurgia, encaminhou-se a paciente para a oncologia clínica, que liberou sem necessidade de quimioterapia ou radioterapia. O retorno se deu de 3 em 3 meses por 1 ano, e após esse prazo o retorno se deu de 6 em 6 meses por 2 anos. Durante esse período, a paciente esteve assintomática e sem sinais de lesões residuais ou recidivas em região cervical. Foi realizada tomografia cervical e tórax de controle, além de ultrassonografia do local primário do tumor e da tireoide sem alterações.

\section{DISCUSSÃO}

No nosso estudo foi relatado um caso clínico de uma paciente com dermatofibrosarcoma protuberante (DFSP), do sexo feminino, com 59 anos. A 
paciente contrapõe a distribuição prevalente do sexo masculino e o pico de idade relatado por outros autores. Esse caso faz com que haja um confronto da literatura quanto ao local acometido; apresentamos um DFSP cervical, sendo menos frequente, pois ao longo do nosso estudo ficou notório que o tronco foi tido como o local mais comum para que haja o DFSP, seguido pelos membros inferiores, membros superiores e por último, cabeça e pescoço.

O tumor pode ser confundido com lesões benignas ou passar despercebido pelo próprio paciente, visto que muitas vezes se trata como lesão inespecífica e assintomática e seu tamanho é relativo ao tempo de desenvolvimento. O DFSP possui baixo risco de metástase, e as suas taxas de reincidir são altas e vinculadas a altas taxas de morbidades, justificando a necessidade do diagnóstico e tratamento precoce para que haja uma maior taxa de cura.

Clinicamente, o diagnóstico se torna difícil caso encontrado de forma precoce, isso se dá devido à diferenciação que possa ocorrer com má formação vascular cutânea. De acordo com o artigo Ressecção alargada no tratamento do dermatofibrossarcoma protuberante (2015), a "dor pode se manifestar em $25 \%$ dos casos. A evolução é caracterizada, inicialmente, por uma fase de placas, que originam um ou mais nódulos, conferindo ao tumor um aspecto protuberante." (ALVES et al., 2015, n.p.).

Constantemente, a neoplasia se coincide a um queloide. Esse tumor pode ser manifestado como: lesão atrófica, o que se assemelharia, à esclerodermia circunscrita. Para que se possa ter uma possível avaliação da extensão do tumor, diante da área em que ele se encontra, é necessário que se faça, exame físico, ressonância magnética e tomografia computadorizada. A principal forma de tratamento, é pela extração completa das células tumorais, preservando ao máximo os tecidos sadios, para que se mantenha a função e aparência estética.

A literatura relata que $3 \mathrm{~cm}$ de margem larga diminui, significativamente, a taxa de recidiva, dessa forma, no caso clínico apresentado, a margem cirúrgica de excisão adotada foi de $3 \mathrm{~cm}$ de tecido sadio, de margens de pele normal para margens macroscópicas e na biópsia para fáscia subjacente. Apesar da cirurgia ser o padrão 
de tratamento, a radioterapia adjuvante se torna efetiva na prevenção do tumor caso haja uma recidiva de DFSP, após a resseção com margens positivas.

No nosso estudo não houve presença de metástases, a excisão foi ampla e sem realização de radioterapia adjuvante com boa evolução. O acompanhamento pós cirúrgico foi realizado em conjunto com os oncologistas do Hospital do Câncer de Mato Grosso. O aumento da incidência pode ser devido aos pacientes estarem educados para reconhecer a desenvoltura do tumor, consequentemente, estão mais conscientes em relação a saúde, e, portanto, busca-se aconselhamento médico para esse tumor.

Figura 1. Dermatofibrossarcoma protuberante.

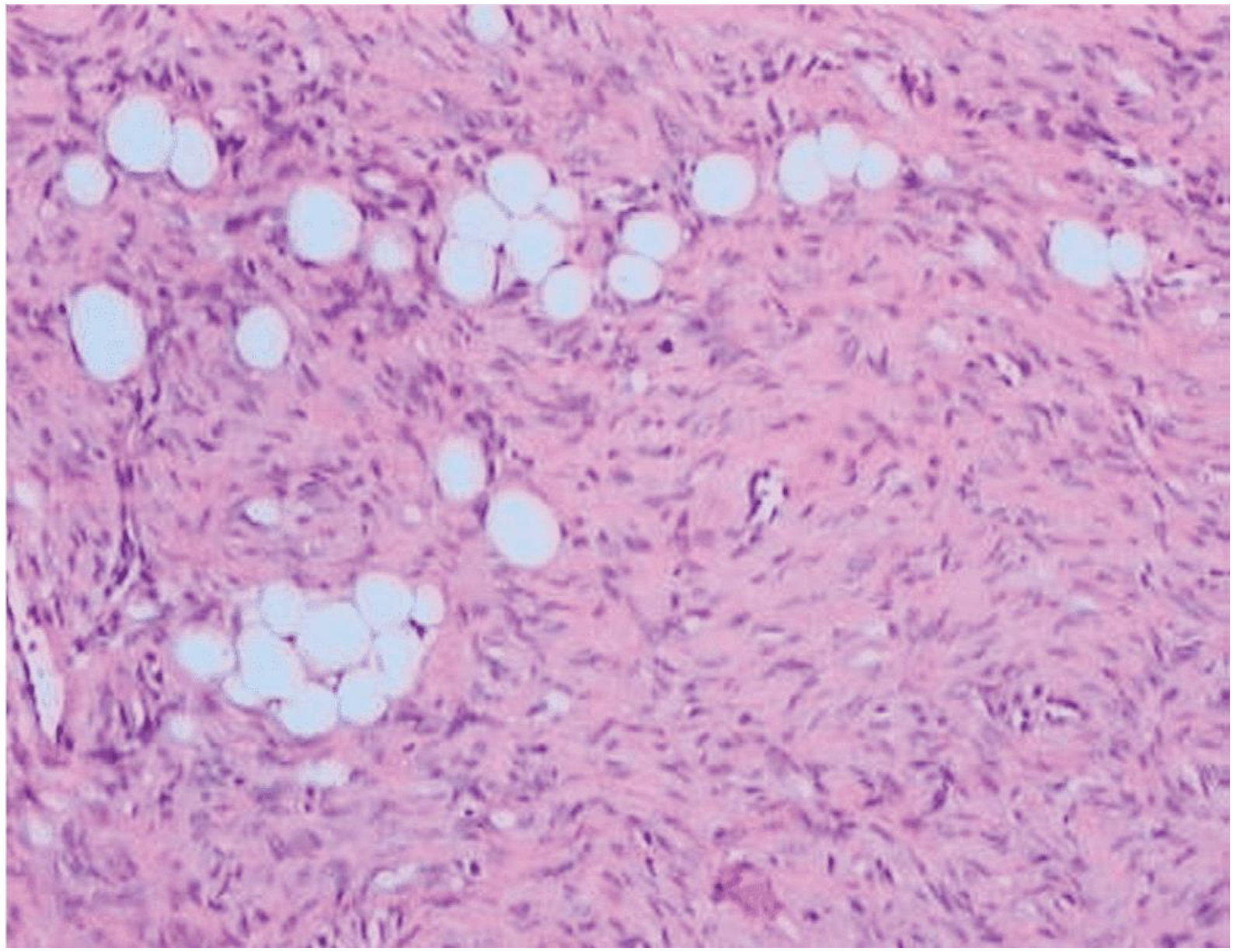

Fonte: Anais Brasileiros de Dermatologia. (2006)

A figura 1 configura-se com padrão microestoriforme envolvendo difusamente o tecido adiposo com aspecto em favo de mel (HE 400x). 
Figura 2. Paciente de 55 anos, sexo feminino.

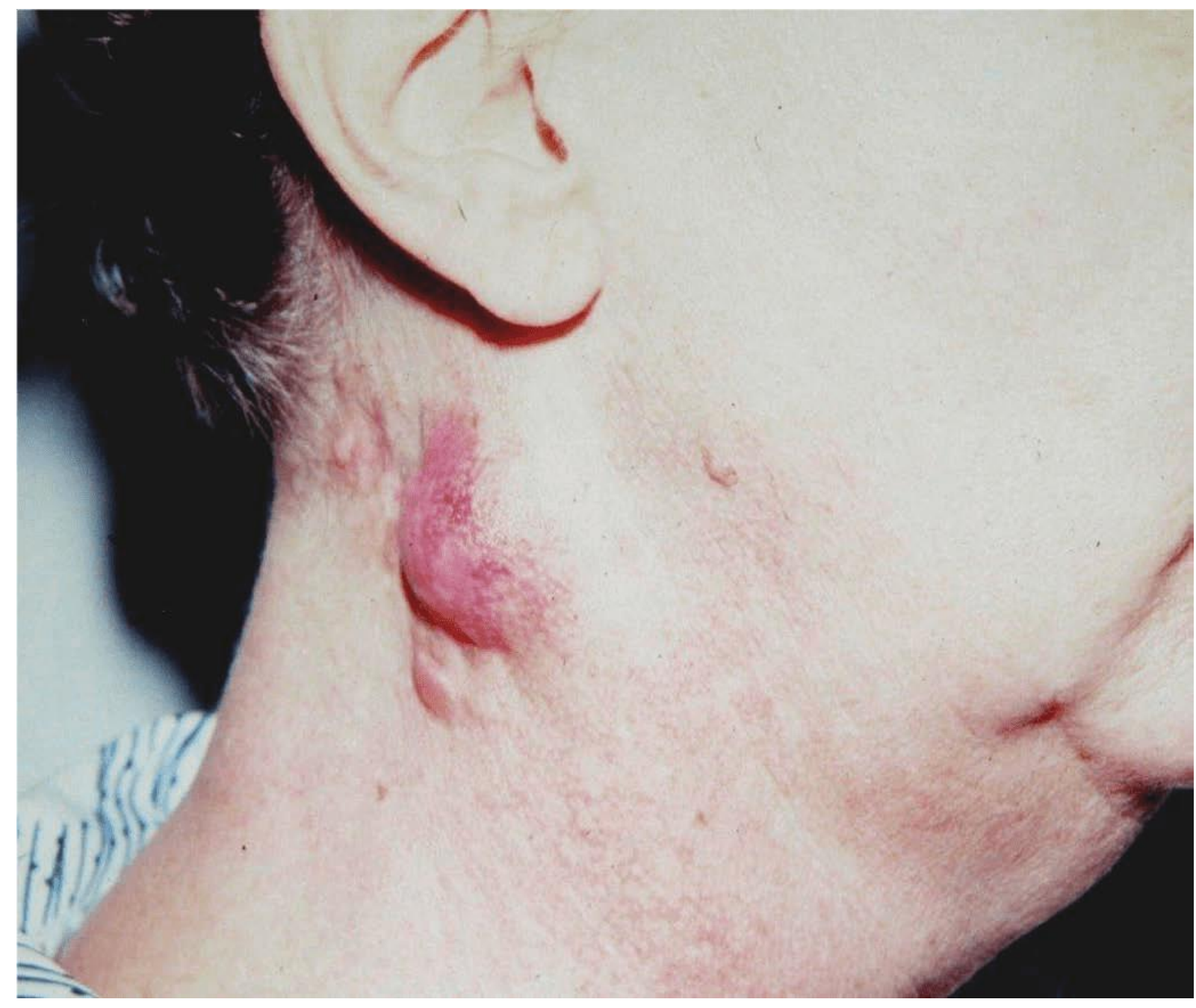

Fonte: Revista Brasileira Cirurgia Plástica. (2016)

A figura 2 se relaciona a lesão em região cervical lateral direita. Nota-se tumefação arredondada e elevada, de cor avermelhada e com telangiectasias. 
Figura 3. Aspecto pós-operatório após 2 meses.

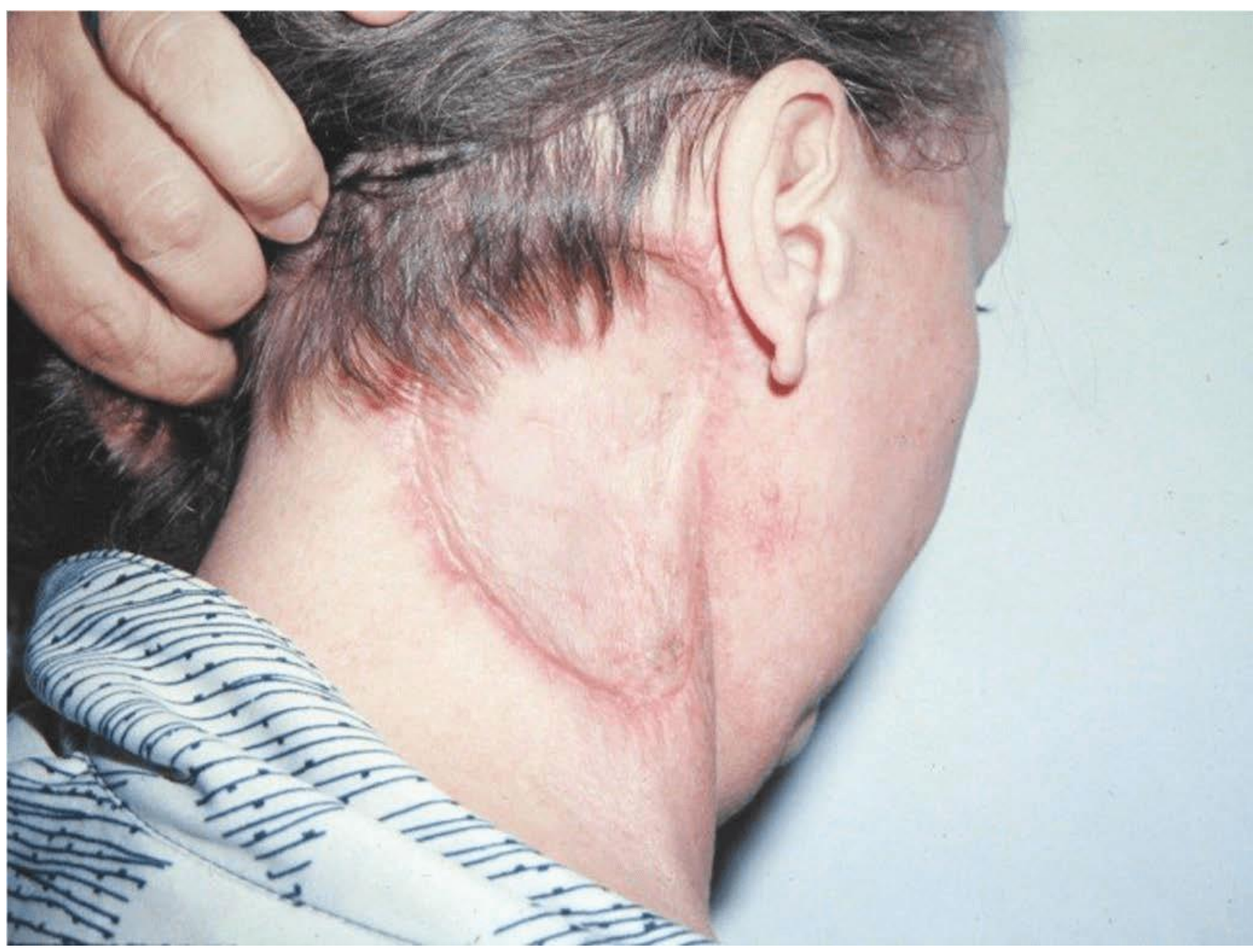

Fonte: Revista Brasileira Cirurgia Plástica (2016)

A figura 3 se atém ao pós-operatório, fazendo um contraponto com a figura 2.

\section{CONSIDERAÇÕES FINAIS}

O dermatofibrosarcoma protuberante é um tumor maligno, que apesar do crescimento lento, pode ser agressivo e recidivante. A terapia cirúrgica apropriada deve ser através de uma excisão com margem ampla e uma ressecção tridimensional, alcançando fáscia profunda. Apresentamos um caso, cujo tumor comprometeu uma região incomum, o pescoço, em uma paciente do sexo feminino e com 59 anos, contrapondo alguns dados literários. Foi realizado o diagnóstico correto, com exames complementares e uma conduta cirúrgica adequada, sem recidiva nos três anos de pós-operatório. Confirmando assim, a eficácia do tratamento até o momento, mesmo 
sem radioterapia adjuvante. Dessa forma, conclui-se a importância do seguimento rigoroso clínico e por imagem no pós-operatório deste tipo de tumor, para que a efetividade da vigilância oncológica seja alcançada.

\section{REFERÊNCIAS}

ALVES, José Carlos Ribeiro Resende; FONSECA, Rebeca Paowha Liu; SILVA, Aloisio Ferreira; ANDRADE, José de Souza; ARAUJO, Izabella Costa; ALMEIDA, Augusto César de Melo; PEREIRA, Nárlei; NEFFA, Lívia. Ressecção alargada no tratamento do dermatofibrossarcoma protuberante. Revista Brasileira Cirurgia Plástica, v. 29, n. 3, p. 395-403, junho. 2014. Disponível em: < http://www.rbcp.org.br/details/1555/pt-BR/resseccao-alargada-no-tratamento-dodermatofibrossarcoma-

protuberante\#: :text=INTRODU\%C3\%87\%C3\%830\%3A\%20Dermatofibrossarcoma \%20Protuberante\%20(DFSP), ressec\%C3\%A7\%C3\%A30\%20alargada\%2C\%20com \%20margens\%20vari\%C3\%A1veis.> Acesso: 22 agosto 2020.

ALVES, José Carlos Ribeiro Resende. Ressecção alargada no tratamento do dermatofibrossarcoma protuberante. Revista Brasileira Cirurgia Plástica, v. 29, n. 3, p. 395-403, junho. 2014. Acesso: 22 agosto 2020.

CAPPELLINA, Cesare; BRAMBULLO, Tito; SALMASO, Roberto; BASSETTO, Franco. Dermatofibrosarcoma protuberans: série de 27 casos consecutivos. Revista Brasileira Cirurgia Plástica, v. 31, n. 2, p. 235-241, abril. 2016. Disponível em: <http://www.rbcp.org.br/details/1740/pt-BR/dermatofibrosarcoma-protuberans--seriede-27-casos-consecutivos> Acesso: 22 agosto 2020.

DINIZ, Michelle dos Santos; RAMOS, Daniel Contijo; NUNES, Mauricio Buzeli. Dermatofibrossarcoma protuberans: localização não usual e a importância da cirurgia micrográfica de Mohs. Surgical And Cosmetic Dermatology, v. 5, n. 2, p. 173-6, junho. 2013. Disponível em: <http://www.surgicalcosmetic.org.br/detalheartigo/273/Dermatofibrossarcoma-protuberans--localizacao-nao-usual-e-aimportancia-da-cirurgia-micrografica-de-Mohs> Acesso: 22 agosto 2020. 
FLEURY JR, Luiz Fernando Fróes; SANCHES JR, José Antônio. Sarcomas cutâneos primários. Anais Brasileiros de Dermatologia, v. 81, n. 3, p. 207-21, 2006. Disponível em:

https://www.scielo.br/scielo.php?script=sci_arttext\&pid=S036505962006000300002> Acesso: 22 agosto 2020.

MACEDO, Jefferson Lessa Soares; BARBOSA, Gustavo Salviano; ROSA, Simone Corrêa. Dermatofibrossarcoma protuberante. Revista Brasileira Cirurgia Plástica, v. $30, \quad$ n. $3, \quad$ p. 506-509, abr/mai. 2015. Disponível em: <http://www.rbcp.org.br/details/1670/pt-BR/dermatofibrossarcoma-protuberante> Acesso: 22 agosto 2020.

MACEDO, Jefferson Lessa Soares; ROSA, Simone Corrêa. Dermatofibrossarcoma protuberante. Revista Brasileira Cirurgia Plástica, v. 23, n. 2, p. 138-43, jan. 2008. $<$ http://www.rbcp.org.br/details/18/dermatofibrosarcoma-

protuberans\#: :text=Dermatofibrossarcoma\%20protuberante\%20\%C3\%A9\%20um\% 20tumor,recorr\%C3\%AAncia\%20local\%20ap\%C3\%B3s\%20excis\%C3\%A30\%20cir \%C3\%BArgica.> Acesso: 22 agosto 2020.

PEREIRA, Thomas Jefferson Reis; FERNANDES, Márcio Costa; REBELO, Vângelis; GOMES, Pedro Henrique; CORREIA, Jéssica; MEDEIROS, Roseana. Dermatofibrossarcoma protuberans em couro cabeludo - Relato de caso e revisão de literatura. Relatos Casos Cirúrgicos, v. 5, n. 1 p. 2022, jan. 2019. Disponível em: < http://relatosdocbc.org.br/detalhes/184/dermatofibrossarcomaprotuberans-em-couro-cabeludo---relato-de-caso-e-revisao-de-literatura> Acesso: 22 agosto 2020.

PORTUGAL, Erick; ALVES, José Carlos Ribeiro; FONSECA, Rebeca Paowha Liu; ANDRADE, José de Souza; ALMEIDA, Augusto César de Melo; ARAÚJO, Izabella Costa; PEREIRA, Nárlei; SILVA, Raíssa. Dermatofibrossarcoma protuberante diagnosticado erroneamente como queloide e tratado com acetonido de triancinolona. Revista Brasileira Cirurgia Plástica, v. 31, n. 1, p. 82-87, jan. 2016. Disponível em: < http://www.rbcp.org.br/details/1715/pt-BR/dermatofibrossarcoma- 
protuberante-diagnosticado-erroneamente-como-queloide-e-tratado-com-acetonidode-triancinolona> Acesso: 22 agosto 2020.

STIVAL, Belissa Cristina; Dermatofibrosarcoma protuberans inicialmente diagnosticado como queloide - Relato de caso. Revista do Médico Residente, v. 4, n. 2, p. 132-135, abr/jun. 2012.

Enviado: Fevereiro, 2020.

Aprovado: Agosto, 2020. 